\title{
Cutaneous adverse reactions in a lung cancer patient treated with pembrolizumab: A case report
}

\author{
CARMEN BOBEICA $^{1 *}$, LAURA REBEGEA ${ }^{2}$, GABRIEL MURARIU $^{3}$, \\ MICHAELA DOBRE ${ }^{1}$, AUREL NECHITA ${ }^{2}$, ALIN LAURENTIU TATU $^{2,4^{*}}$, \\ ELENA NICULET $^{1,5}$, LUCRETIA ANGHEL $^{2}$, SILVIA FOTEA $^{2 *}$ and MIHAELA CRAESCU ${ }^{1,6}$ \\ ${ }^{1}$ Department of Morphological and Functional Sciences, ${ }^{2}$ Clinical Medical Department, Faculty of Medicine and Pharmacy, \\ 'Dunarea de Jos' University, 800010 Galati; ${ }^{3}$ Department of Chemistry, Physics and Environment, Faculty of Sciences \\ and Environment, 'Dunarea de Jos' University, 800201 Galati; ${ }^{4}$ Research Center in The Field of Medical \\ and Pharmaceutical Sciences, ReFORM-UDJ, 'Dunarea de Jos' University, 800010 Galati; \\ Departments of ${ }^{5}$ Pathology and ${ }^{6}$ Clinical Radiotherapy, 'Sfantul Apostol Andrei' \\ Emergency Clinical Hospital, 800578 Galati, Romania
}

Received August 6, 2021; Accepted September 6, 2021

DOI: $10.3892 /$ etm.2021.10937

\begin{abstract}
Lung cancer is the main cause of oncological death in the US and worldwide, constituting a significant public health problem. The incidence of lung cancer is on the increase. In the present study, the diagnostic process was carried out and treatment options were considered to determine the therapeutic response of a patient diagnosed with lung cancer. The case of an early stage lung cancer patient who benefited from surgical treatment was presented. The pathology report stated the complete diagnosis to be pleomorphic lung cancer
\end{abstract}

Correspondence to: Dr Elena Niculet, Department of Morphological and Functional Sciences, Faculty of Medicine and Pharmacy, 'Dunarea de Jos' University, 35 Alexandru Ioan Cuza Street, 800010 Galati, Romania

E-mail: helena_badiu@yahoo.com

Professor Gabriel Murariu, Department of Chemistry, Physics and Environment, Faculty of Sciences and Environment, 'Dunarea de Jos' University, 111 Domneasca Street, 800201 Galati, Romania E-mail: gabriel_murariu@yahoo.com

*Contributed equally

Abbreviations: ALK, anaplastic lymphoma kinase; CT, computerized tomography; ECOG, Eastern Cooperative Oncology Group; EGFR, epidermal growth factor; FDA, Food and Drug Administration; LC, lung cancer; MRI, magnetic resonance imaging; NSCLC, non-small cell lung cancer; PD-1, programmed cell death protein-1; PD-L1, programmed death ligand-1; RTE, radiotherapy; TD, total dose; TP53, tumor protein p53; TTF1, thyroid transcription factor 1; USA, United States of America

Key words: lung cancer, therapeutic strategy, pembrolizumab, cutaneous adverse effects, PD-L1 with an adenocarcinoma component, pT2aNOM0, with focal positivity for thyroid transcription factor 1 (TTF1), without epidermal growth factor receptor (EGFR) mutations and ALK recombinations, having an initial clinical stage of IB and programmed death ligand-1 (PD-L1) positivity with a tumor proportion score of over $70 \%$. The patient underwent radiotherapy treatment and was administered osteoclast inhibitors and immunotherapy, with no favorable therapeutic effect and with the presence of secondary cutaneous adverse effects to pembrolizumab. As a main cause of death, lung cancer registers a low general survival rate even in patients with targeted therapies or immunotherapy. By better identifying the patients at risk, one can establish a more efficient personalized treatment; the future objective of scientific studies is the follow-up of adverse effects of new therapies.

\section{Introduction}

Lung cancer (LC), the main cause of oncological death in the US and globally, is a significant public health problem. The incidence of LC is on the rise, much as in neuroendocrine tumor or gastric carcinoma cases $(1,2)$. In general, LC is a highly aggressive form of cancer with a rapid rate of metastasis $(3,4)$.

In recent decades, LC has become the most frequent form of cancer worldwide, with a possible IL-6 involvement through its pro-angiogenic properties, which helps in cancer development and/or progression. The number of new cases was estimated at 1,8 million in $2012(3,5,6)$. As the leading cause of oncological death, with a poor prognosis, LC has one of the lowest 5-year survival rates at under $15 \%(7,8)$.

The aim of the current study was to present the case of a patient and the cutaneous side effects attributed to pembrolizumab therapy. The outcome of the current study revealed that pembrolizumab immune therapy use managed to prolong the patient's survival with about 4 months, having a good performance status of 2 (ECOG). 


\section{Case report}

Subject. The aim of the current study was to present a case of a patient and the cutaneous side effects attributed to pembrolizumab therapy. A 63-year-old patient was admitted in March 2017 to the Military Hospital of Galați, Romania, suffering from right thoracic stabbing pain, dyspnea, dry cough and night sweats. The patient signed and provided written informed consent for the publication of data or any images, which is available in the patient's medical chart. Ethics approval and consent to participate were obtained from the 'Sfantul Apostol Andrei' Emergency Clinical Hospital's Ethics Committee, with the decision no. 11413 from 03.06.2021.

Computerized tomography scan. After a thoracic computerized tomography (CT) scan in September 2017, a tumor mass in the right superior pulmonary lobe with a background of diffuse moderate pulmonary emphysema was found.

In October 2017, the patient was subjected to a surgical procedure consisting of right superior lobectomy and mediastinal lymphadenectomy in the 'Marius Nasta' Institute of Pneumophysiology, in Bucharest, Romania. The pathology laboratory reported this tumor as a pleomorphic lung cancer with an adenocarcinoma component, pT2aNOM0, with focal positivity for thyroid transcription factor 1 (TTF1), without epidermal growth factor receptor (EGFR) mutations, nor anaplastic lymphoma kinase (ALK) recombinations, having an initial clinical stage of IB and programmed death ligand-1 (PD-L1) positivity with a tumor proportion score of over $70 \%$.

A new thoracic CT scan was performed in March 2018 which revealed a right apex lung tumor with spine involvement and mediastinal paratracheal ipsilateral adenopathies (Fig. 1A and B).

A skeletal scintigraphy was carried out at the end of March 2018 which revealed multiple bone metastases localized in the head of the humerus, the T3 vertebra, right third rib and right iliac bone, for which the patient received a bone tissue protection treatment with zolendronic acid until September 2018 and also antalgic radiotherapy (RTE) with a total dose (TD) of $20 \mathrm{~Gy} / 5 \mathrm{fr}$ localized at the head of the humerus, right sacroiliac region and the thoracic spine, levels $\mathrm{T} 1$ to T4. No systemic anticancer treatment was administered from July 2018 until September 2019.

The CT scan of the thorax and abdomen, which was performed in September 2018, revealed a right apex tumor mass measuring 65/50/45 $\mathrm{mm}$, which invaded the thoracic wall and destroyed the $2 \mathrm{nd}$, 3rd and 4th posterior rib arcs. The tumor protruded from the dorsal medullary canal, as a local, continuous tumor evolvement. In November 2018, the magnetic resonance imaging (MRI) of the thoracic spine highlighted the right lung tumor mass which destroyed the thoracic vertebral bodies of $\mathrm{T} 2$ to $\mathrm{T} 4$, and which invaded the intervertebral pedicles on the right side and also the spinal canal. Consequently, the patient underwent a surgical procedure for spinal decompression and the pathology report revealed that the fibro-hyaline fragments of the vertebral discs had foci of poorly differentiated squamous cell carcinoma (G3).
Other factors and treatment. At the same time, the laboratory findings revealed high values for urea and creatinine; the decision for terminating treatment with zolendronic acid was taken, in favor of a systemic one, such as chemotherapy or immunotherapy. Due to the high, oscillating creatinine values, it was possible to administer chemotherapy based on carboplatin in association with pemetrexed, paclitaxel or gemcitabine.

The recent inclusion of pembrolizumab in the national cancer treatment program, the patient's ALK and EGFR statuses, his PD-L1 level of $70 \%$, and that this drug is excluded from treatment only in cases with severe renal insufficiency, supported the initiation of first-line immunotherapy with pembrolizumab starting from September 2018. Two months after therapy with pembrolizumab was begun, a CT scan of the head, thorax and abdomen was carried out and it revealed an evolutionary stable disease. Immunotherapy was continued but some eczematous, psoriasiform and lichenoid secondary cutaneous side effects developed, which were localized on the left mandible and left superior limb and had a partial response to corticoid treatment with full resolution following pembrolizumab treatment termination (Figs. 2 and 3) (9-12).

The patient suffered from arterial hypertension for which he received metoprolol, daily. An important fact that is well known among physicians is that metoprolol should not be concomitantly administered with floctafenine, sultopride, bepridil, diltiazem or verapamil, nor should it be taken during or after a meal, as food has the property of increasing the bioavailability of metoprolol. The types of food that are ingested are important, not only in the case of metoprolol, but also for other medications such as statins (grapefruit, for example, has the ability to increase the drug's blood concentration). There are also other factors that can intervene in certain drug pharmacokinetics, as well as physiological factors such as decreased fatty tissue or gastric acidity, or decreased renal excretion. Diuretics (hydrochlorothiazide), and other anti-hypertensive drugs, can also have cutaneous adverse reactions including rashes or photosensitization. The cutaneous adverse reactions of some B-blockers have been reported, such as psoriasis precipitation or exacerbation, but fortunately our patient did not suffer from this disease (13-19).

Due to the cutaneous adverse reaction development, dexamethasone was prescribed, as $8 \mathrm{mg}$ injectable doses, 2 vials per day, for 5 days, which relieved the patient of the cutaneous lesions, initially attenuating them, followed by complete extinction. Thus, treatment with pembrolizumab could be reinstated and continued.

The patient developed a mediastinal compression syndrome (Fig. 4) for which mediastinal radiotherapy was administered with a TD of $30 \mathrm{~Gy}$. Immunotherapy was continued in the subsequent months and was terminated by March 2019, after a thoracic CT scan was carried out which revealed progressive disease (Fig. 5A and B).

The patient received 4 doses of pembrolizumab, having a current unsatisfactory general health status with a prognosis index of 3 on the Eastern Cooperative Oncology Group performance status (ECOG). Consequently, immunotherapy was stopped. 


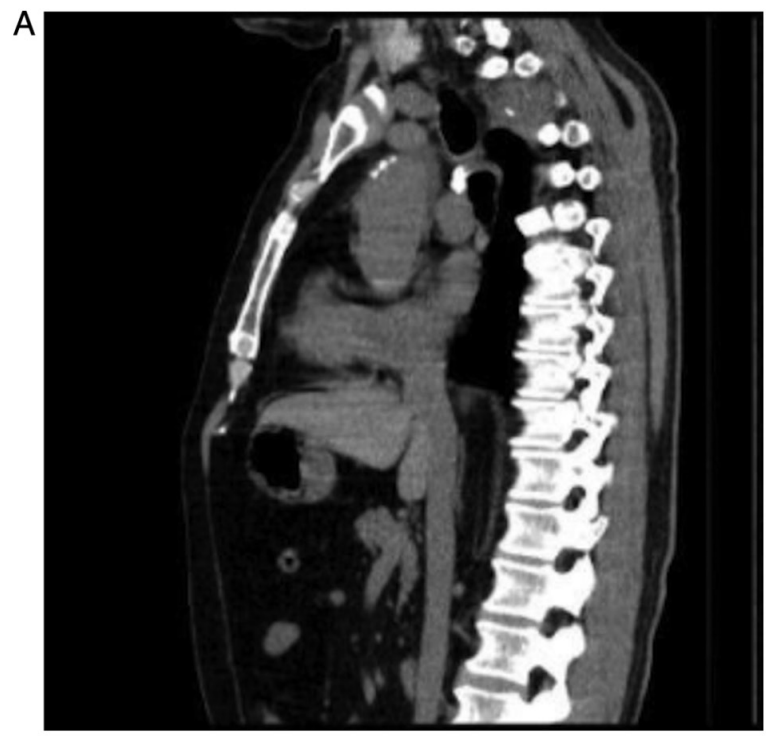

B

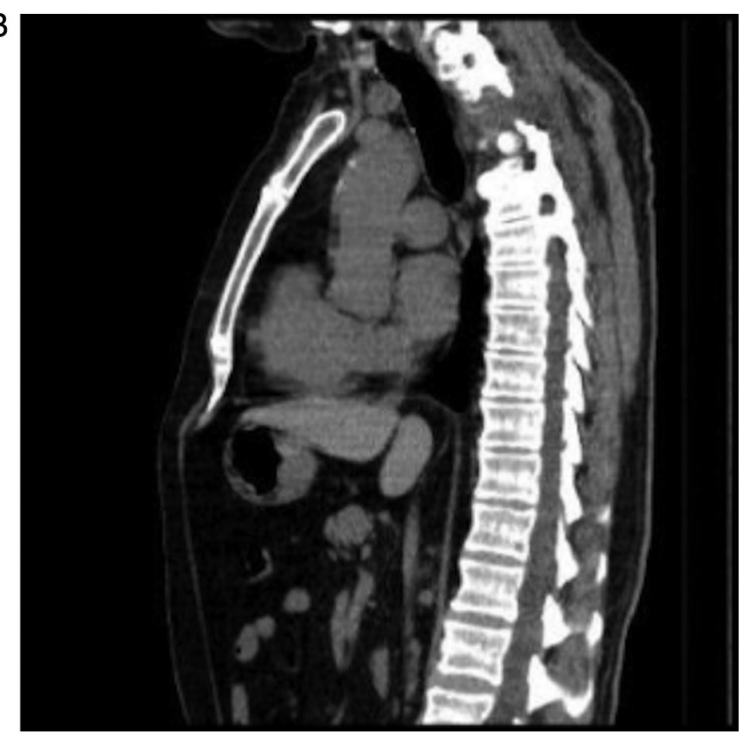

Figure 1. (A and B) Thoracic CT scan. Right lung apex tumor with spine involvement and mediastinal paratracheal ipsilateral adenopathies. CT, computerized tomography.

\section{Discussion}

Adenocarcinoma is the most frequent pathology subtype, registering an increased incidence rate among female patients in industrialized countries over the last decade, a tendency justified by some authors through a change in toxic habits, with a higher use of filtered cigarettes, leading to deeper inhalation with a more peripheral distribution $(20,21)$. According to the new diagnostic and treatment guides, surgical intervention, radiotherapy, adjuvant chemotherapy, targeted therapy and immunotherapy are employed as therapeutic options in non-small cell lung cancer (NSCLC) with the possibility of being used as monotherapy or in combination, in accordance with the stage of disease. Although early diagnosis enables a complete surgical resection (the therapeutic option with the highest potential for cure), approximately $40 \%$ of patients relapse 5 years after the procedure $(4,22,23)$.

As part of the NSCLC treatment, new compounds have been introduced, targeting immune control points, such as

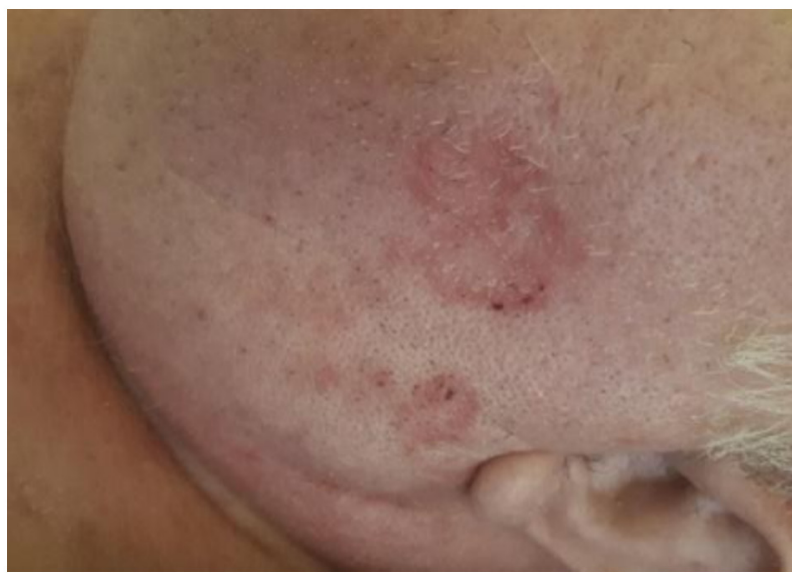

Figure 2. Secondary cutaneous adverse effects localized on the left mandible.

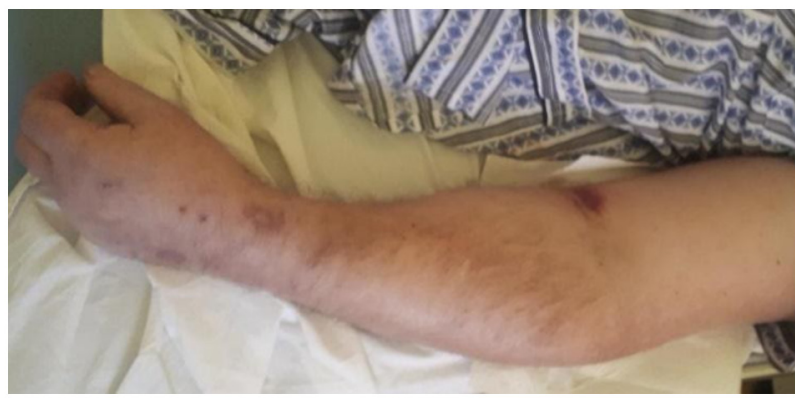

Figure 3. Secondary cutaneous adverse effects localized on the superior limb.

programmed cell death protein-1 (PD-1) or its ligand (PD-L1). Pembrolizumab is one such immunotherapeutic anti-PD-1 recommended for treating advanced-stage patients, without EGFR and ALK mutations, and with high PD-L1 $(4,24)$. Tumor protein p53 (TP53) and EGFR mutations are strong parameters that can predict the response to anti-PD-1 treatment in NSCLC $(25,26)$.

Pembrolizumab was approved by the Food and Drug Administration (FDA) in the USA for many advanced stage or metastatic cancers as this therapeutic agent acts by blocking the protein found on the surface of cancer cells, and the protein known as PD-L1, thus allowing immune cells to destroy the tumor. Recent findings suggest that treatment with pembrolizumab can help some NSCLC patients benefit from a higher survival rate with fewer adverse effects. The phase I clinical study known as KEYNOTE-001 proved that some advanced stage NSCLC patients who received pembrolizumab lived 3-4 times longer than expected. The most frequent cutaneous adverse effects identified were brief cutaneous eruptions which our patient developed since the second month of treatment with this medication (27-29).

The latest literature states that cases with mild cutaneous adverse reactions (such as lichenoid reactions, granulomatous skin reactions), as in our patient's case, can receive treatment with topical corticosteroids at low doses, moisturizing ointments or oral antihistamines $(27,28,30,31)$.

Some patients who developed cutaneous adverse reactions such as vitiligo registered skin repigmentation, not as proof for skin lesion treatment response, but as proof for cancer 


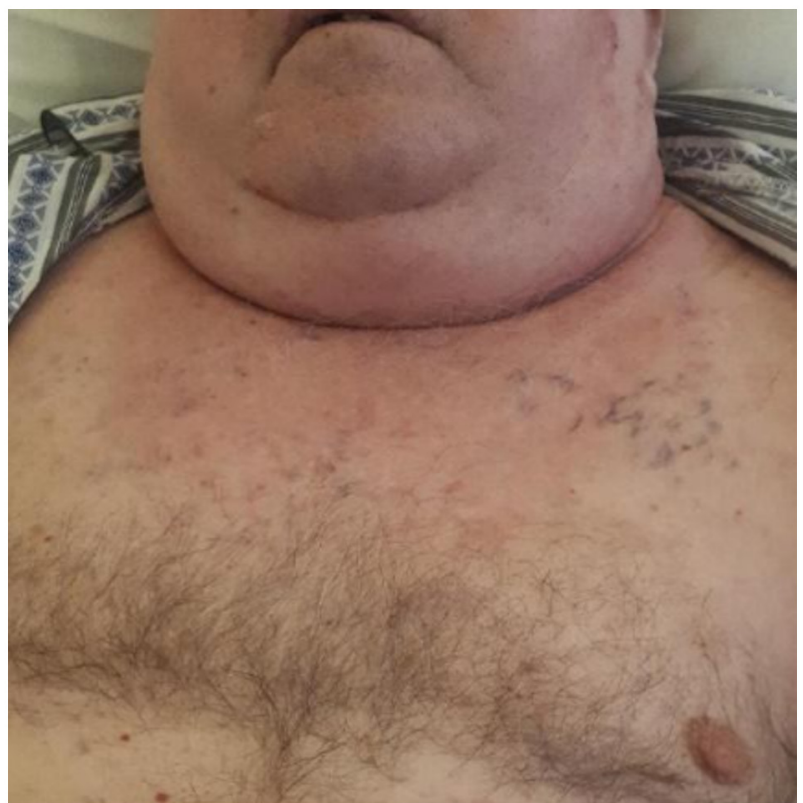

Figure 4. Mediastinal compression syndrome.

(melanoma) progression. The skin lesions were treated with classical vitiligo treatment, i.e., sun protection, phototherapy, calcineurin inhibitors, and topical corticosteroids. Psoriasis is a frequent adverse reaction to pembrolizumab and can develop as de novo lesions or as an exacerbation of the pre-existing lesions. Therapeutic management in these cases included topical steroids and/or vitamin D analogues, systemic retinoids, or even methotrexate. Cases of pityriasis rubra pilaris have also been reported after pembrolizumab therapy (some also as a paraneoplastic syndrome), these patients being successfully treated with acitretin and topical steroids (27-31).

More severe cutaneous adverse reactions (extensive bullous pemphigoid lesions) were treated with topical and systemic corticosteroids and treatment with pembrolizumab was withheld (as a temporary therapeutic management approach, or even as a permanent one; as in some patients with lupus erythematosus, toxic epidermal necrolysis, Stevens-Johnson syndrome or erythema multiforme). These adverse effects can evolve in various directions: they can resolve completely, can be ongoing, or they can become exacerbated. Some of the patients suffering from such therapeutic events, even after withholding pembrolizumab treatment, had a partial therapeutic response regarding their skin lesions $(27,28,32)$.

An important issue to further research concerns the group of pembrolizumab drug interactions. For instance, one case report presented the case of a patient treated both with pembrolizumab and rivaroxaban, who developed an intra-cerebellar hemorrhage; as pembrolizumab seems to have an effect on the liver's CYP3/A4 system, it also seems to influence the metabolism of such new anticoagulant medication (33).

Notably, the cutaneous adverse effects treatment must not interfere with the therapeutic effects of pembrolizumab (anti-PD1) and patients need to adhere to treatment; such patients can opt for a dose decrease or can even stop treatment. Skin lesions being highly visible, the patients suffer from a low quality of life, with psychological distress and social
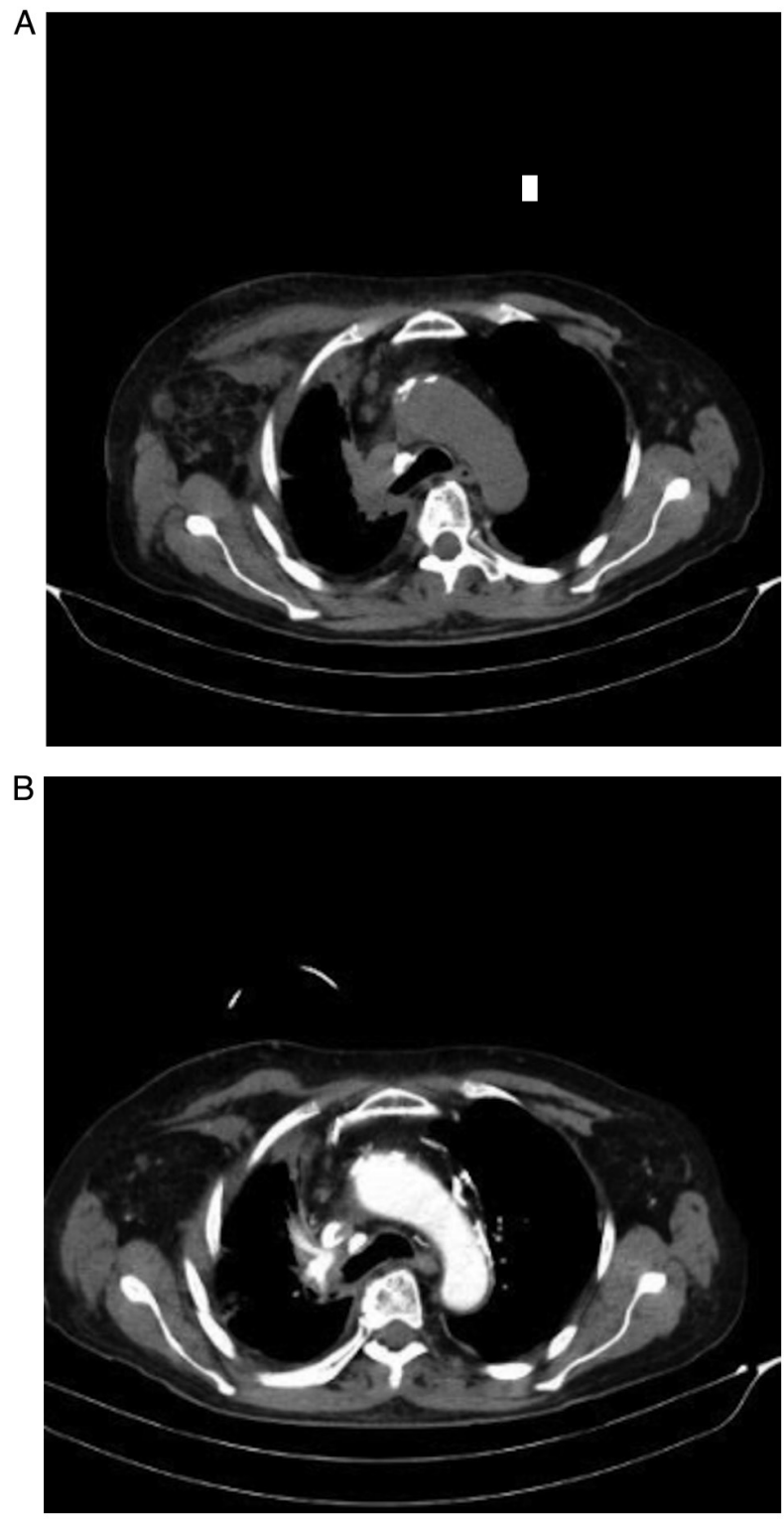

Figure 5. (A and B) CT of the thorax. Progressive disease is evident. CT, computerized tomography.

refrain, similar to those with systemic sclerosis, for example. Topical steroids have been used as treatment for such adverse effects, having beneficial results, mostly due to their multiple biologic activities, which include anti-inflammatory, immune suppressive, or anti-proliferative activities. Complete pembrolizumab treatment withdrawal was found in rare cases in the literature, more often clinicians opting for continuation of treatment or only for a temporary withdrawal (for 1 or even 7 weeks) $(27,28,33-36)$.

During clinical trials, patients who develop severe adverse reactions to immunotherapy (such as pembrolizumab) are not allowed to resume it, due to the high risk of reoccurrence. This is also an in-practice issue, as the choice to reinitiate immune therapy is challenging. One possible approach would involve a class switch, opting for an anti-CTLA-4, instead of an anti-PD(L)1 (pembrolizumab), these two having different mechanisms of action (the first one increases the diversity of the host's immune response, while the second reactivates 
a suppressed host immune response). Another approach to patient treatment is the re-challenge itself, as many patients experience no recurrence of the side effects. Reintroducing the same anti-PD1 treatment can also result in some of the patients experiencing the same cutaneous adverse reactions, or some even new ones. Recurrence of the side effects may occur with decreased frequency (37-39).

In summary, even with targeted therapy or immunotherapy, lung cancer remains the main cause of death worldwide, having an increasing incidence in the last decade with a low general survival rate. It is mandatory to find a more favorable approach to identify patients at risk in order to establish more efficient personalized treatment and part of this is identifying the adverse effects of new therapies, as an objective for future studies.

Although immunotherapy is one of the newly reached frontiers in cancer therapy, clinicians need to have a watchful eye for (cutaneous) adverse reactions, their early diagnosis and management having a major impact on patient treatment concerning adherence, therapeutic result and overall survival. Treatment must be adapted to each patient, as they can react differently to pembrolizumab therapy, with various side effects.

\section{Acknowledgements}

The current work was academically supported by the 'Dunarea de Jos' University of Galati, Romania, through the research center - Multidisciplinary Integrated Center of Dermatological Interface Research (MIC-DIR) [Centrul Integrat Multidisciplinar de Cercetare de Interfata Dermatologica (CIM-CID)].

\section{Funding}

No funding was received.

\section{Availability of data and materials}

The information generated and analyzed during the current study is available from the corresponding author on reasonable request.

\section{Authors' contributions}

CB, GM, ALT, SF, EN, MC, LA, LR, MD and AN were major contributors in writing the manuscript; they were involved in all the stages of the study, contributed to the conception and design of the work, as well as revising it; they helped analyze the data for the work, revised it for important intellectual content and approved the final version to be published. All authors agreed to be accountable for all aspects of the work in ensuring that questions related to the accuracy or integrity of any part of the work. All authors have had equal participation, contribution and equal rights to this article. All authors read and approved the final manuscript.

\section{Ethics approval and consent to participate}

Ethics approval and consent to participate were obtained from the 'Sfantul Apostol Andrei' Emergency Clinical Hospital's Ethics Committee, with the decision no. 11413 from 03.06.2021.

\section{Patient consent for publication}

The patient provided written informed consent for the publication of any associated data and accompanying image.

\section{Competing interests}

The authors declare that they have no competing interests.

\section{References}

1. Craescu M, Rebegea L, Ivan I, Dumitru M, Serban C and Firescu D: Therapeutic challenges in a case of trachea neuroendocrine tumor. Acta Med Mediterr 35: 1493-1496, 2019.

2. Fekete GL, Cotoi OS and Fekete JE: Multiple nodular cutaneous metastases as the first clinical sign of signet ring cell gastric carcinoma: Case report. Acta Dermatovenerol Croat 20: 34-37, 2012.

3. Riihimäki M, Hemminki A, Fallah M, Thomsen H, Sundquist K, Sundquist J and Hemminki K: Metastatic sites and survival in lung cancer. Lung Cancer 86: 78-84, 2014.

4. National Comprehensive Cancer Network (NCCN): NCCN Clinical Practice Guidelines in Oncology (NCCN guidelines): Non-small cell lung cancer. Version 5.2019 - June 7, 2019. NCCN, Plymouth Meeting, PA, 2019. https://www.nccn.org/guidelines/ category_1\#si. Accessed July 30, 2019.

5. Nomori $\bar{H}$, Watanabe K, Ohtusuka T, Naruke T, Suemasu K and Uno K: The size of metastatic foci and lymph nodes yielding false-negative and false-positive lymph node staging with positron emission tomography in patients with lung cancer. J Thorac Cardiovasc Surg 127: 1087-1092, 2004.

6. Niculet E, Chioncel V, Elisei AM, Miulescu M, Buzia OD, Nwabudike LC, Craescu M, Draganescu M, Bujoreanu F, Marinescu E, et al: Multifactorial expression of IL-6 with update on COVID-19 and the therapeutic strategies of its blockade (Review). Exp Ther Med 21: 263, 2021.

7. Dela Cruz CS, Tanoue LT and Matthay RA: Lung cancer: Epidemiology, etiology and prevention. Clin Chest Med 32: 605-644, 2011 .

8. Zhou H, Wu A, Fu W, Lv Z and Zhang Z: Significance of semaphorin-3A and MMP-14 protein expression in non-small cell lung cancer. Oncol Lett 7: 1395-1400, 2014.

9. Gheorghe I, Tatu AL, Lupu I, Thamer O, Cotar AI, Pircalabioru GG, Popa M, Cristea VC, Lazar V and Chifiriuc MC: Molecular characterization of virulence and resistance features in Staphylococcus aureus clinical strains isolated from cutaneous lesions in patients with drug adverse reactions. Rom Biotech Lett 22: 12321-12327, 2017.

10. Hwang SJE and Fernández-Peñas P: Adverse reactions to biologics: Melanoma (Ipilimumab, Nivolumab, Pembrolizumab). Curr Probl Dermatol 53: 82-92, 2018.

11. Lee M and Seetharamu N: An atypical presentation of lichen planus-like reaction from pembrolizumab. Case Rep Dermatol Med 2019: 4065437, 2019.

12. Nwabudike LC and Tatu AL: Reply to Gambichler T et al: Altered epigenetic pathways and cell cycle dysregulation in healthy appearing skin of patients with koebnerized squamous cell carcinomas following skin surgery. J Eur Acad Dermatol Venereol 33: e3-e4, 2019.

13. Flaten $\mathrm{HK}$ and Monte AA: The pharmacogenomic and metabolomic predictors of ACE inhibitor and angiotensin II receptor blocker effectiveness and safety. Cardiovasc Drugs Ther 31: 471-482, 2017.

14. Johnson JA: Advancing management of hypertension through pharmacogenomics. Ann Med 44 (Suppl 1): S17-S22, 2012.

15. Tatu AL, Elisei AM, Chioncel V, Miulescu M and Nwabudike LC: Immunologic adverse reactions of $\beta$-blockers and the skin (Review). Exp Ther Med 18: 955-959, 2019.

16. Nwabudike LC, Elisei AM, Buzia OD, Miulescu M and Tatu AL: Statins. A review on structural perspectives, adverse reactions and relations with non-melanoma skin cancer. Rev Chim (Bucharest) 69: 2557-2562, 2018.

17. Tatu AL, Ciobotaru OR, Miulescu M, Buzia OD, Elisei AM, Mardare N, Diaconu C, Robu S and Nwabudike LC: Hydrochlorothiazide: Chemical structure, therapeutic, phototoxic and carcinogenetic effects in dermatology. Rev Chim (Bucharest) 69: 2110-2114, 2018. 
18. Dobre M, Georgescu C, Stefanescu V, Cuciureanu M, Nechita A and Arbune M: Homeostatic changes during anticonvulsant medication in children. Farmacia 63: 402-406, 2015.

19. Jáuregui-Garrido B and Jáuregui-Lobera I: Interactions between antihypertensive drugs and food. Nutr Hosp 27: 1866-1875, 2012

20. Fidler-Benaoudia MM, Torre LA, Bray F, Ferlay J and Jemal A: Lung cancer incidence in young women vs. young men: A systematic analysis in 40 countries. Int J Cancer 147: 811-819, 2020.

21. Özmen S and Ceylan O: Trends in lung cancer incidence within the last 10 years: An Eastern Anatolian single center experience. J Surg Med 4: 112-115, 2020.

22. Blandino G and Di Agostino S: New therapeutic strategies to treat human cancers expressing mutant p53 proteins. J Exp Clin Cancer Res 37: 30, 2018.

23. NSCLC Meta-analyses Collaborative Group; Arriagada R, Auperin A, Burdett S, Higgins JP, Johnson DH, Le Chevalier T, Le Pechoux C, Parmar MK, Pignon JP, et al: Adjuvant chemotherapy, with or without postoperative radiotherapy, in operable non-small-cell lung cancer: Two meta-analyses of individual patient data. Lancet 375: 1267-1277, 2010.

24. Sui H, Ma N, Wang Y, Li H, Liu X, Su Y and Yang J: Anti-PD-1/PD-L1 therapy for non-small-cell lung cancer: Toward personalized medicine and combination strategies. J Immunol Res 2018: 6984948, 2018.

25. Biton J, Mansuet-Lupo A, Pécuchet N, Alifano M, Ouakrim H, Arrondeau J, Boudou-Rouquette P, Goldwasser F, Leroy K, Goc J, et al: TP53, STK11, and EGFR mutations predict tumor immune profile and the response to anti-PD-1 in lung adenocarcinoma. Clin Cancer Res 24: 5710-5723, 2018.

26. WuCHand Hwang MJ: Risk stratification forlung adenocarcinoma on EGFR and TP53 mutation status, chemotherapy, and PD-L1 immunotherapy. Cancer Med 8: 5850-5861, 2019.

27. Sanlorenzo M, Vujic I, Daud A, Algazi A, Gubens M, Luna SA Lin K, Quaglino P, Rappersberger K and Ortiz-Urda S: Pembrolizumab cutaneous adverse events and their association with disease progression. JAMA Dermatol 151: 1206-1212, 2015.

28. Simonsen AB, Kaae J, Ellebaek E, Svane IM and Zachariae C: Cutaneous adverse reactions to anti-PD-1 treatment-a systematic review. J Am Acad Dermatol 83: 1415-1424, 2020.

29. Garon EB, Rizvi NA, Hui R, Leighl N, Balmanoukian AS, Eder JP, Patnaik A, Aggarwal C, Gubens M, Horn L, et al: Pembrolizumab for the treatment of non-small-cell lung cancer. N Engl J Med 372: 2018-2028, 2015.
30. Fekete GL, Boda D, Căruntu C and Fekete L: Paraneoplastic pityriasis rubra pilaris in association with prostate carcinoma: A case report and literature review. Exp Ther Med 18: 5052-5055, 2019.

31. Coleman E, Panse G, Haldas J, Gettinger SN and Leventhal JS: Pityriasis rubra pilaris-like erythroderma in the setting of pembrolizumab therapy responsive to acitretin. JAAD Case Rep 4: 669-671, 2018

32. Creţu A, Dimitriu A, Brănişteanu D and Brinişteanu DE: Erythema multiforme-etiopathogenic, clinical and therapeutic aspects. Rev Med Chir Soc Med Nat Iasi 119: 55-61, 2015.

33. Joshi N (ed): Pembrolizumab/rivaroxaban interaction. In: Reactions Weekly. Vol 1704. Springer Nature Switzerland AG, Cham, p304, 2018.

34. Bobeică C, Tatu AL, Crăescu M and Solovăstru L: Dynamics of digital ulcers in systemic sclerosis. Exp Ther Med 20: 61-67, 2020.

35. Bobeica C, Niculet E, Craescu M, Onisor C, Bujoreanu F, Draganescu ML, Halip IA and Gheuca-Solovastru L: Epidemiological profile of systemic sclerosis in the southeast region of Romania. Exp Ther Med 21: 77, 2021.

36. Niculet E, Bobeica $C$ and Tatu AL: Glucocorticoid-induced skin atrophy: The old and the new. Clin Cosmet Investig Dermatol 13: 1041-1050, 2020

37. Haanen J, Ernstoff M, Wang Y, Menzies A, Puzanov I, Grivas P, Larkin J, Peters S, Thompson J and Obeid M: Rechallenge patients with immune checkpoint inhibitors following severe immune-related adverse events: Review of the literature and suggested prophylactic strategy. J Immunother Cancer 8: e000604, 2020

38. Winer A, Bodor JN and Borghaei H: Identifying and managing the adverse effects of immune checkpoint blockade. J Thorac Dis 10 (Suppl 3): S480-S489, 2018.

39. Bhatlapenumarthi V, Patwari A and Harb AJ: Immune-related adverse events and immune checkpoint inhibitor tolerance on rechallenge in patients with irAEs: A single-center experience. J Cancer Res Clin Oncol 147: 2789-2800, 2021

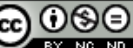

This work is licensed under a Creative Commons Attribution-NonCommercial-NoDerivatives 4.0 International (CC BY-NC-ND 4.0) License. 